\title{
Does the Motor System Need Intermittent Control?
}

\author{
Ian David Loram¹, Cornelis van de Kamp ${ }^{1}$, Martin Lakie², Henrik Gollee ${ }^{3}$, and Peter J. Gawthrop ${ }^{3,4}$ \\ ${ }^{1}$ Cognitive Motor Function Research Group, School of Healthcare Science, Manchester Metropolitan University, \\ Manchester; ${ }^{2}$ School of Sport and Exercise Science, University of Birmingham, Birmingham; and ${ }^{3}$ School of \\ Engineering, University of Glasgow, Glasgow, United Kingdom; and ${ }^{4}$ Melbourne School of Engineering, \\ University of Melbourne, Melbourne, Victoria, Australia.
}

LORAM, I.D., C. VAN DE KAMP, M. LAKIE, H. GOLLEE, and P.J. GAWTHROP. Does the motor system need intermittent control? Exerc. Sport Sci. Rev., Vol. 42, No. 3, pp. 117-125, 2014. Explanation of motor control is dominated by continuous neurophysiological pathways (e.g., transcortical, spinal) and the continuous control paradigm. Using new theoretical development, methodology, and evidence, we propose intermittent control, which incorporates a serial ballistic process within the main feedback loop, provides a more general and more accurate paradigm necessary to explain attributes highly advantageous for competitive survival and performance. Key Words: intermittent control, sensorimotor control, selection, adaptation, psychological refractory period

\section{INTRODUCTION}

\section{Attributes of Human Movement}

For humans, movement is fundamental to quality of life, and the attributes of natural biological movement distinguish it from artificial attempts at replication. The term "robotic," when applied to humans, still indicates unnatural rigid behavior. The majority of human motor control including balance, locomotion, postural, and manual control is learned during childhood and later life. Response-stimulus associations, experienced even only once or accidentally, potentially are stored within the nervous system as possibilities for motor response (29). Environmental stimuli generally activate multiple possibilities for action from which motor responses are selected (3). Depending on the outcome, selections are reinforced progressively or down-weighted $(4,7,35)$. Neurophysiological mechanisms facilitate two kinds of control, each with their own merits (Fig 1A). Fast pathways implement selections that, when sufficiently facilitated, can be triggered without online analysis of the consequences of the response. These are habitual responses, described as reflexive because causality is environmental (using

Address for correspondence: Ian David Loram, Ph.D., Cognitive Motor Function Research Group, School of Healthcare Science, Manchester Metropolitan University, John Dalton Bldg, Oxford Road, Manchester, M1 5GD, United Kingdom (E-mail: i.loram@mmu.ac.uk).

Accepted for publication: April 2, 2014

Associate Editor: Roger M. Enoka, Ph.D. preselected choices) (35). Slow pathways implement intentional control in which the causality is more internal (using online analysis and selection) $(22,35)$.

Throughout biological history, harsh conditions of prey and predation have given a competitive edge to individuals who are original, able to produce unpredictable movement, and explore new possibilities during current environmental conditions (2). Consistent with biological evolution (2), human movement has inherited a capacity for robustness, variety, flexibility, and adaptability made possible by exploiting the many kinematic muscle activation and control strategy degrees of freedom available through our neuromuscular mechanisms. In sport, exercise, and performance, humans define themselves by developing and testing attributes of strength, endurance, speed, flexibility, and dexterity. Our view, shared by others (1), is that the most advantageous attribute is the ability to construct and implement new motor solutions in the moment of activity $(1,2)$. An open question is the control paradigm and neurophysiological mechanisms that explain these flexible attributes of human motor control.

\section{The Explanatory Power of the Continuous Control Paradigm}

The control of movement depends on sensory feedback to regulate performance. The automatic feedback servo-mechanism has inspired the neurophysiology of reflexes dating from Sherrington. The dominant paradigm today is still continuous control (Fig. 1B) (27,31). During the 1960s, Kleinman et al. (16), investigating the behavior of humans in the control loop of high-performance machines, demonstrated that the mean human response to stimuli was well explained by a continuous 


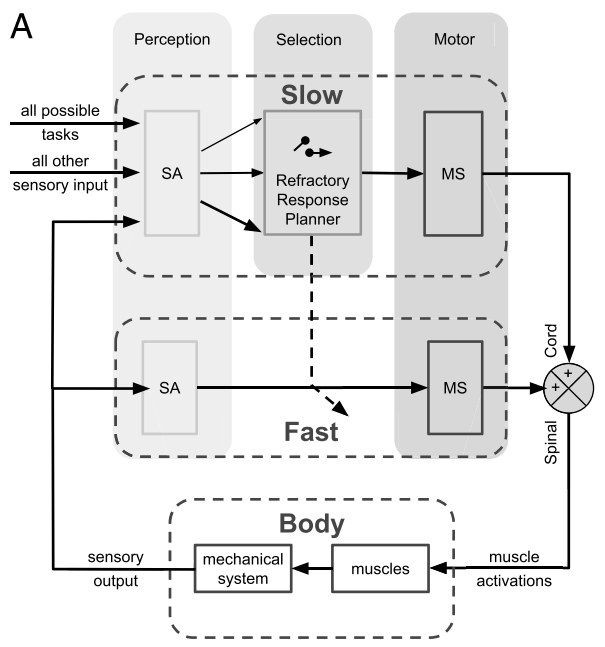

B

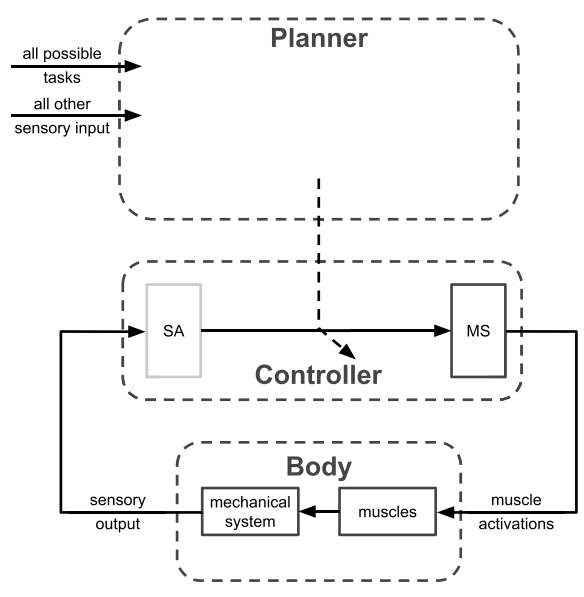

C

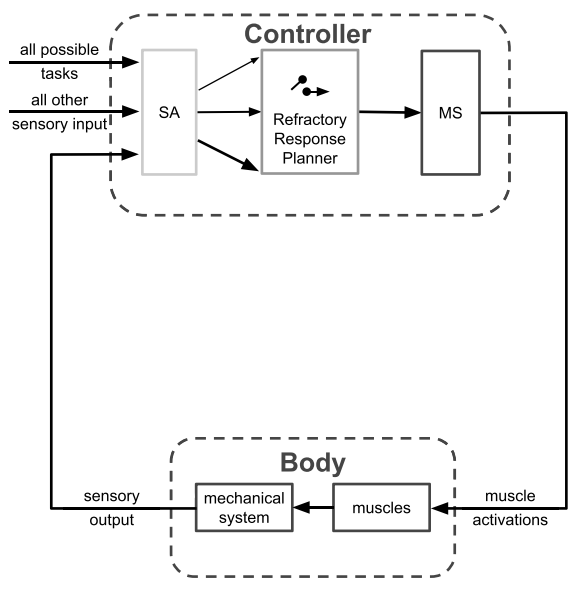

Cortical loops

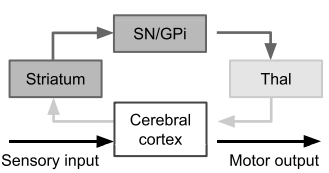

Subcortical loops

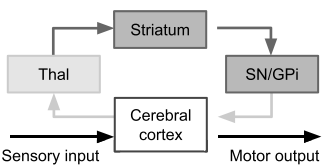

Figure 1. Continuous and intermittent control. A. General overall scheme integrating continuous and intermittent control. The perceptual process of sensory analysis (SA) integrates all sensory modalities with prior experience. Selection of goals, control priorities, actions, and submovements occurs in the Refractory Response Planner. Selection converges to a serial process with a maximum rate of two to four selections per second: the proposed selection pathway includes the basal ganglia loops $(4,7,13,15,28)$. The motor system (MS) translates selected goals, actions, and submovements into coordinated motor output using the machinery of action representations, motor primitives, motor modules, pattern generators, muscle modes, and synergies (4,7,32). Within a slow intermittent feedback loop restricted to the voluntary bandwidth $(1-2 \mathrm{~Hz})$, the motor system generates coordinated motor responses sequentially from each new selection. Within the fast continuous loop restricted to a higher bandwidth (>10 Hz) acting through transcortical, brain stem, and spinal pathways, the motor system uses selected parameters to modulate habitual-reflexive feedback $(20,33)$. Refractoriness distinguishes the slow from the fast loop. In this serial process, refractoriness is the increased delay in selecting and forming one response before the previous process has completed (33). B. Continuous control hypothesis (100\% fast pathway): task selection occurs at the higher Planner level ordering the selected goal and control law to be used continuously via the low-level feedback mechanism. This feedback loop consists of the "Controller" enclosing the continuous stages of SA and MS. C. Intermittent control hypothesis (100\% slow pathway): the Refractory Response Planner forms an intermediate stage between SA and MS. The refractory response planner decides when to implement a new response and provides time within the feedback loop for selecting and optimizing the control law that will be used to construct the open-loop control trajectory. D. Cortical and subcortical sensorimotor loops through the basal ganglia (28). Cortical-loops: For corticobasal ganglia loops, the position of the thalamic relay is on the return arm of the loop. Subcortical loops: In the case of all subcortical loops, the position of the thalamic relay is on the input side of the loop. Predominantly excitatory regions and connections are shown in gray, whereas inhibitory regions and connections are black. Thal indicates thalamus; SN/GP, substantia nigra/globus pallidus. The fast pathway (A, B) corresponds to the lower direct route between Sensory input and Motor output. The slow pathway (A, C) corresponds to the upper loop through the basal ganglia.

controller with a Kalman filter (optimal state estimator) and optimal predictor. Moreover, by adding suitably filtered random noise to the motor and sensory states, he could fit perfectly by calculating the variability in human control (18).

Subsequently, ideas of optimal state estimation (e.g., Bayesian integration), prediction and optimal coordination have been applied in explanation of human control $(27,31)$. However, we suggest that paradigmatic advance in explanation since Kleinman has been limited.

The continuous control paradigm finds its natural counterpart in the high bandwidth spinal, brain stem, and transcortical feedback pathways (27) represented as the fast feedback loop in Figure 1A and Figure 1B and lowermost pathway in Figure 1D. Much accumulated evidence summarized beautifully in (27) demonstrates the power and sophistication of transcortical responses that are a class of fast-acting responses of latency $(\sim 60-120 \mathrm{~ms})$ triggered by environmental stimuli. Within the posterior parietal cortex, this pathway integrates proprioceptive, visual, audio, cutaneous, and vestibular sensations, with expectations generated from motor output passing from the cerebellum $(14,27)$. Including input from the frontal cortex, these responses are modulated by preceding factors including explicit external instructions and the implicit behavioral context including the current posture and task goals and by the external environment including the direction of the gravitational-acceleration vector and location of objects (27). Transcortical responses include habitual responses. These are learned responses that are facilitated sufficiently that integrated 
sensory input triggers motor responses transcortically, bypassing the slow loop through the basal ganglia (Fig. 1A, D) $(4,7,35)$. Given a certain environmental and task context, prior intent can only alter the scale (magnitude) of the fast response (22). Within its duration, there is no evaluation of its predicted outcome and no possibility of selecting different or opposite responses $(4,7,35)$.

These fast responses can be modeled within the continuous control paradigm in which control signals are calculated continuously from the current estimated state of the system using a precomputed control law $(5,27,31)$. The mathematics of optimal control provides a control law that best satisfies the task goal and cost function given the physical plant being controlled. This hierarchical paradigm (Fig. 1B), in which task level goals influence fast continuous feedback loops, is in principle appropriate for modulating the complex mapping of multiple sensory inputs to multiple motor outputs organized in a functional modular manner including synergies, motor primitives, and pattern generators $(31,32)$. This paradigm of continuous feedback using an optimal control law currently is dominant in explaining upper limb control, balance, and posture $(5,31)$. Textbooks on motor control provide much detail on the mechanisms that facilitate the fast component to human motor control for achieving temporary goals and for maintaining sustained set points. Detail on the slow pathway (Fig. 1A, C) is conspicuous by its absence.

\section{The Hypothesis of Intermittent Control}

Intermittent control (IC) (Fig. 1C) is a general control paradigm in which sensory feedback is used intermittently to parameterize control trajectories $(8,11,30)$. IC is shown as the slow feedback loop in Figure 1A and Figure 1C. Control is executed as a sequence of open-loop trajectories, that is, without modification by sensory feedback apart from the instances of intermittent feedback. IC contains a single-channel serial ballistic process, the refractory response planner within the slow feedback loop (Fig. 1A, C). Serial ballistic means that control proceeds as a sequential process in which control trajectories are planned using current sensory information and executed open loop. We use the terms "serial ballistic control" and "intermittent control" interchangeably. The duration for which the serial process is unresponsive to sensory input is known as the psychological refractory period (26).

If a continuous feedback control law could be formulated in advance to provide the necessary relationship between the current state of the system and control trajectories, the current environmental stimuli and the current response then planning outside the loop would be appropriate (30). However, if the goal, structure, or constraints of the control are dependent on system states, this prior computation is not possible and the selection and optimization process must occur within the feedback loop (30).

The feature distinguishing intermittent from continuous control is the open loop interval. Within this interval, the control trajectory is open loop but is time varying continuously in an optimal manner (30). Unlike continuous control, intermittent control provides the time required to plan and select the control trajectory within the feedback loop (Fig. 1C) rather than outside the feedback loop (Fig. 1B) (30). The length of the intermittent interval gives a trade-off between continuous control (zero intermittent interval) and intermittency. Continuous control maximizes the frequency bandwidth and stability margins at the cost of reduced flexibility, whereas intermittent control allows in the loop optimization and selection (32) at the cost of reduced frequency bandwidth and reduced stability margins.

Increased computational time within the feedback loop is useful when control solutions are required to comply with external constraints, internal constraints, and system properties that are temporary, not easily predictable, or which require state-dependent solutions (30). It takes time to resolve uncertainty (noise) in motor and sensory signals and uncertainty between competing motor responses of differing consequences $(4,7,24,32,33)$. If an unexpected force perturbation occurs, or if pain occurs, or if an obstacle is presented, the appropriate goal, control structure, control law, and limits on joint rotations may depend on the current configuration (state) of the body. The solution requires time for appropriate selection and time for a multivariable optimization using knowledge of the current system state. When the flexibility needed is more than tuning parameters in the currently selected fast solution, it is more economical to select and optimize solutions as required rather than precompute and retrievably store solutions for every eventuality. The rationale for intermittent control is that it confers online flexibility and adaptability that has a biological advantage for performance and survival.

\section{The Need for an Adequate Methodology to Test the Hypothesis}

A general scheme includes continuous and intermittent feedback (Fig. 1A). Our hypothesis, perhaps controversial, is that human motor control is fundamentally intermittent rather than continuous (i.e., the fast loop contributes relatively little power to the summated response). Figure $1 \mathrm{C}$ presents the hypothesis where the continuous pathway has zero weight. This hypothesis refers to task-related control at the whole-system level rather than local control of individual joints. Motor control may or may not be intermittent; however, in the absence of an adequate methodology to test the hypothesis, this question has been open since it was first proposed (34). The methodological problem lies in demonstrating that ongoing control is sequentially open loop even when the control trajectory is smooth and when frequency analysis shows no evidence of regular sampling.

We have proposed that nonvisual pedal control of an inverted pendulum load (21) and quiet postural balance, with or without vision (23), can each be explained as a sequential serial ballistic process. We have shown that, during unperturbed standing balance, muscle adjustments occur at a central rate of 2 to 3 per second, which corresponds to the known psychological refractory period $(26,34)$ and the rate of serial ballistic control actions originally proposed in manual tracking (34). We have tested whether the central rate of balance adjustments changes with the number and combinations of sensory modality (17). We also have tested whether the central rate of adjustments changes when the unstable time constant of the manually controlled load decreases, requiring an increased rate of adjustment to sustain control (19). Both experiments confirm the constancy of this rate, implying it is a preferred or intrinsically limited rate of the 
human controller rather than a property of the closed loop system, including human controller and external load. We also have shown that visually guided control of disturbed second-order but not first-order systems has a feedback time delay of 180 to $230 \mathrm{~ms}$, which is consistent with intentional (>150 ms) rather than habitual reflexive mechanisms (60-120 ms) (22). Unpredictable second-order systems require a longer duration of selection/processing within the loop than first-order systems. Although supportive of the intermittent control hypothesis, this evidence, as has been known since the 1960s, can be reproduced by the continuous control paradigm with suitably tuned parameters and suitably filtered additive noise $(16,18)$. Thus, this earlier evidence is circumstantial rather than direct. Rigorous resolution of this hypothesis has needed the development of a new theory, a new methodology, and new evidence.

\section{NEW CONTROL THEORY AND NEW METHODOLOGY}

In the control-engineering literature, intermittent control was proposed to provide a solution enabling online in-theloop optimization during concurrent control of time-varying systems with time-varying constraints (30). The theory has been advanced subsequently to derive the frequency domain properties of intermittent control (10) and an event-driven version of intermittent control (11). This hybrid control paradigm combines continuous-time and event-driven control $(8,9,11)$. State estimation is continuous, allowing an Event Trigger to decide when to reconstruct, whereas a Generalized Hold determines how (the underlying control basis) to construct the control trajectories $(9,11)$. The Event Trigger uses two rules: (i) a minimum open-loop interval should have elapsed - this defines the maximum rate of triggering and (ii) the error in the predicted state exceeds a threshold. Continuous control is included as the special case when the minimum open loop interval and threshold are both zero. After a triggered event, the state estimate fed to the Generalized Hold is updated. Using the "system matched hold basis" (8), the Generalized Hold generates optimal continuous control output, assuming control is proceeding continuously in the absence of unknown disturbances. When unknown disturbances occur, prediction error accumulates until the threshold is exceeded and the state estimate fed to the Generalized Hold is updated. The new theory shows why it is easy to explain behavior as continuous, even when the mechanism is intermittent, because within the time and frequency domains, IC can masquerade as continuous control $(8,10,12)$.

This new theory has facilitated the development of new methods for investigating the serial ballistic hypothesis $(8,12,24)$. It is now possible to test whether human control is compatible with intermittent control through frequency analysis (12).
A

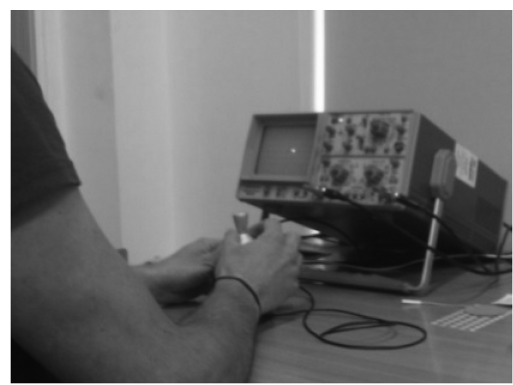

B

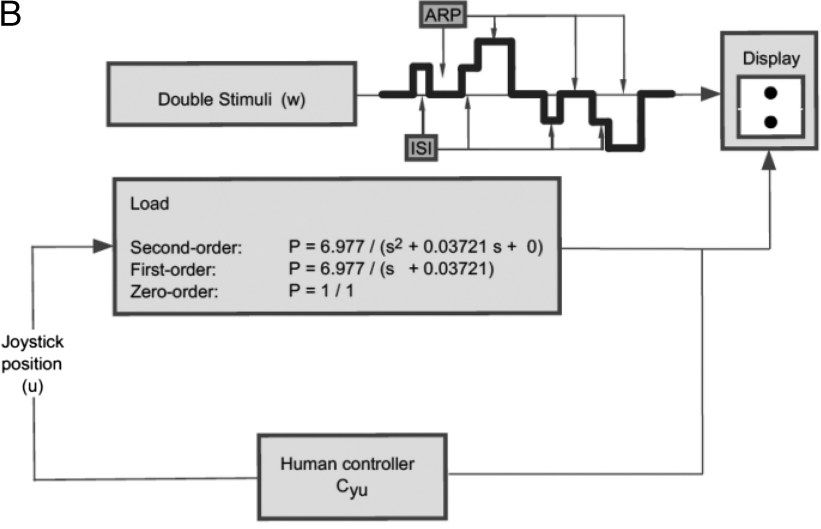

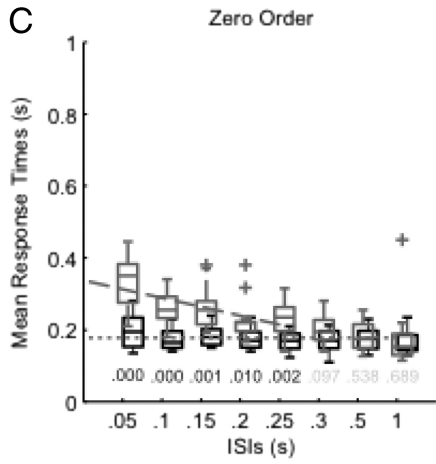
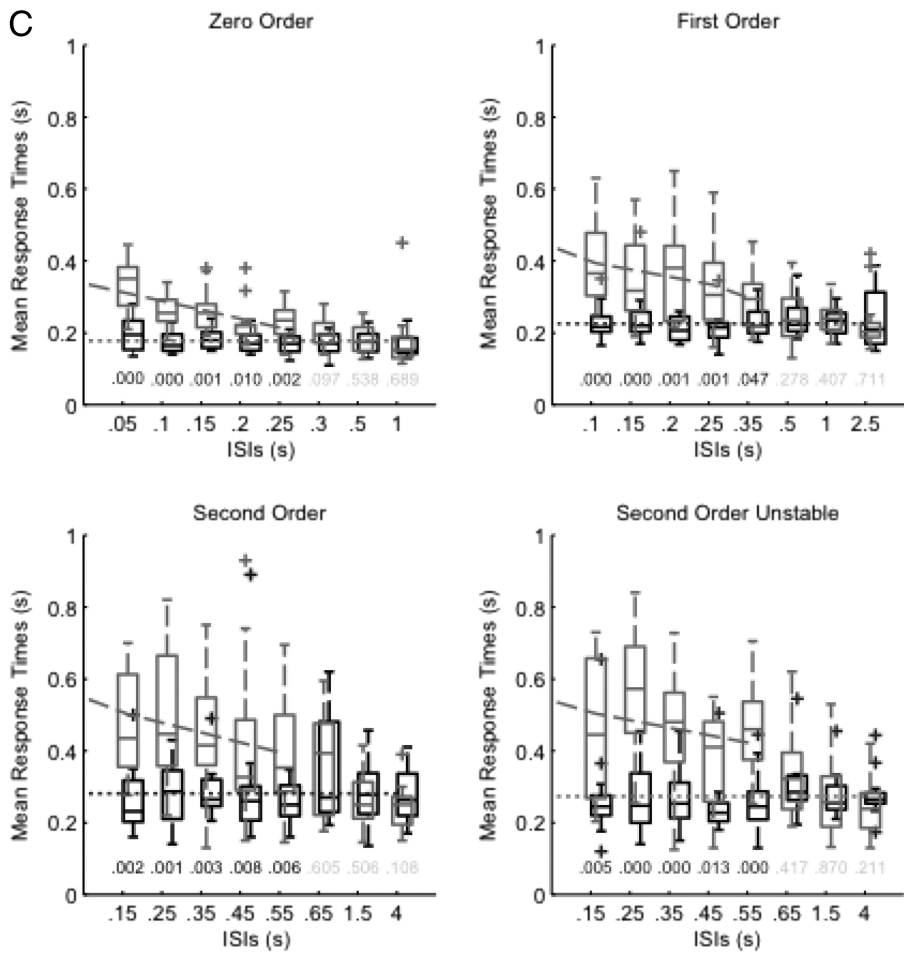

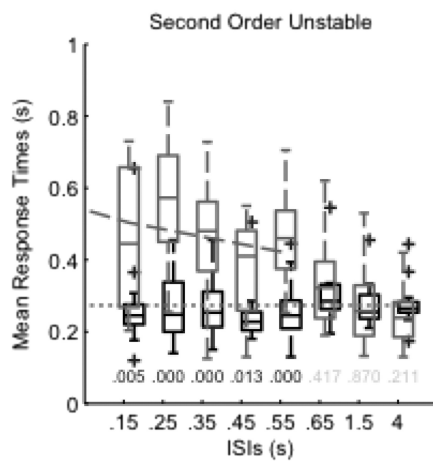

Figure 2. Refractoriness in manual control. A. Task setup. An oscilloscope showed real-time system output position as a small focused dot with negligible delay. Participants provided input to the system using a sensitive, uniaxial, contactless joystick. The system ran in Simulink Real-Time Windows Target within the MATLAB environment (Math-Works). B. Control system and experimental setup. Participants were provided with a tracking target in addition to system output. The tracking signal was constructed from four possible patterns of step sequence (unidirectional and reversed directional step to the left or to the right). First and second stimuli are separated by an unpredictable interstep interval (ISI), patterns are separated by an unpredictable approximate recovery period (ARP). The participant was only aware of an unpredictable sequence of steps. C. Group results (33). The four panels: Zero Order, First Order, Second Order, Second Order Unstable show the interparticipant mean first (RT1, black) and second (RT2, gray) response times against ISI. Values of $P$ of the ANOVA post hoc test are displayed above each ISI level (dark if significant, light if not). [Adapted from (33). Copyright (C) 2013 the authors. Used with permission.] 
Crucially, during sustained control, it also is now possible to discriminate intermittent from continuous control using a time-domain methodology testing for refractoriness (24).

\section{NEW EVIDENCE}

Initially, we have investigated visually guided manual control (Fig. 2A), deliberately excluding proprioceptive and vestibular feedback. If such control is shown to be intermittent, it is worth proceeding to tasks where continuous mechanisms contribute more strongly to motor control.

\section{Refractoriness in Sustained Manual Control}

Using a uniaxial, sensitive, contactless joystick, participants were asked to control four external systems (zero-, first-, and second-order stable and second-order unstable) using visual feedback to track as fast and accurately as possible a target that changes position discretely and unpredictably in time and direction (Fig. 2A, B) (33). For the zero-, first-, and second-order systems, joystick position determines system output position, velocity and acceleration respectively. The unstable second-order system had a time-constant equivalent to a standing human. Because the zero-order system has no dynamics requiring ongoing control, step changes in target produce discrete responses, that is, sharp responses clearly separated from periods of no response. The first- and secondorder systems require sustained ongoing control of the system output position: thus, the step stimuli test responsiveness during ongoing control. The method of analysis, described in detail elsewhere (24), estimates the response time to each step change in target position. The method works by estimating the equivalent (time adjusted) set-point sequence, which has a best linear time invariant relationship between target and joystick (or system output) signals (24). Response times are evaluated in relation to the interstep interval (ISI), which is the duration between the current and the preceding step. Refractoriness (open-loop duration) is indicated by the ISI at which response times diverge between first and second steps (Fig. 2C). A sampling delay (observation delay) is indicated by the ISI at which the enhanced second step response time is maximal. The 13 participants showed evidence of substantial refractoriness, which increased with system order $(0.2-0.5 \mathrm{~s}$; Fig. 2C). For first- and second-order systems, participants showed evidence of a sampling delay $(0.2-0.25 \mathrm{~s})$. This evidence of refractoriness discriminates against continuous control because refractoriness is neither implemented nor explained within the continuous control paradigm. Refractoriness is well explained within the intermittent control paradigm using a corresponding open-loop interval (0.2-0.5 s).

\section{Intermittent Control Explains the Low Bandwidth of Intentional Control}

Using the same setup (Fig. 2A), 11 participants controlled a second-order unstable system during which a multi-sine disturbance was added to their joystick signal (Fig. 3A). We compared control using continuous contact of the joystick with control by gentle taps. The advantage of using taps is, first, that control is explicitly serial ballistic because sensory observation cannot influence control of the system when the hand is not in contact with the joystick and, second, we know the time of individual events (ballistic actions). In both manual conditions, three levels of instruction were used (Fig. 3): keep the dot close to the center, keep the dot still but it does not matter where, and wait as long as possible before controlling the dot. The successfulness of tapping control demonstrated clearly that continuous contact and thus continuous control are not necessary to control an unstable second-order system with dynamics equivalent to a standing human (20). When participants were asked to control position or velocity as closely as possible, they adopted a modal rate of approximately two taps per second (Fig. 3C). This result is important for three reasons. First, the rate is consistent with the refractory durations previously described. Second, the rate is consistent with that observed for muscle adjustments during unperturbed quiet standing (23) and for adjustments during manual control of balance $(17,19)$. Third, a sampling rate of two to four per second explains the lack of coherence between disturbance and joystick beyond approximately 1 to $2 \mathrm{~Hz}$ (Fig. 3B, D). There is a trade-off between rate of control actions and accuracy, which leads to an optimum preferred rate of action. To produce their best performance, we conclude that participants tap at the maximum rate, limited by their refractory duration (open-loop interval) required for the control of this second-order system.

Returning to the continuous contact condition, Figure 3B shows that tapping and continuous contact conditions show the same limited bandwidth of coherence. Frequency analysis shows that the continuous contact condition is compatible with intermittent as well as predictive continuous control, which illustrates the ability of intermittent control to masquerade as continuous control (12). Including the evidence of refractoriness, which continuous control does not explain (Fig. 2), intermittent control provides a more complete explanation of manual control in these tasks.

\section{Refractoriness in Whole-Body Control}

Control of the hand muscles may be more refined, specialized, and more intentional than control of the muscles serving the legs and trunk. Using online visual feedback $(<100$-ms delay) of a marker on the head, participants were asked to track as fast and accurately as possible a target that changes position discretely and unpredictably in time and direction (Fig. 4A). This required head movements of $2 \mathrm{~cm}$ along the anterior-posterior axis, and while participants were instructed not to move their feet, no other constraints or strategies were requested. The eight participants showed evidence of substantial refractoriness ( $\sim 0.5 \mathrm{~s})$ and a sampling delay ( 0.3 s) (Fig. 4B). Refractoriness is not explained within the continuous control paradigm and is explained naturally within the intermittent control paradigm. This result extends the serial ballistic process from control of the hand to integrated intentional control of the whole body (32).

\section{DISCUSSION}

\section{Summary of New Evidence}

Our hypothesis is that human motor control is fundamentally intermittent (Fig. 1C) rather than continuous (Fig. 1B), 

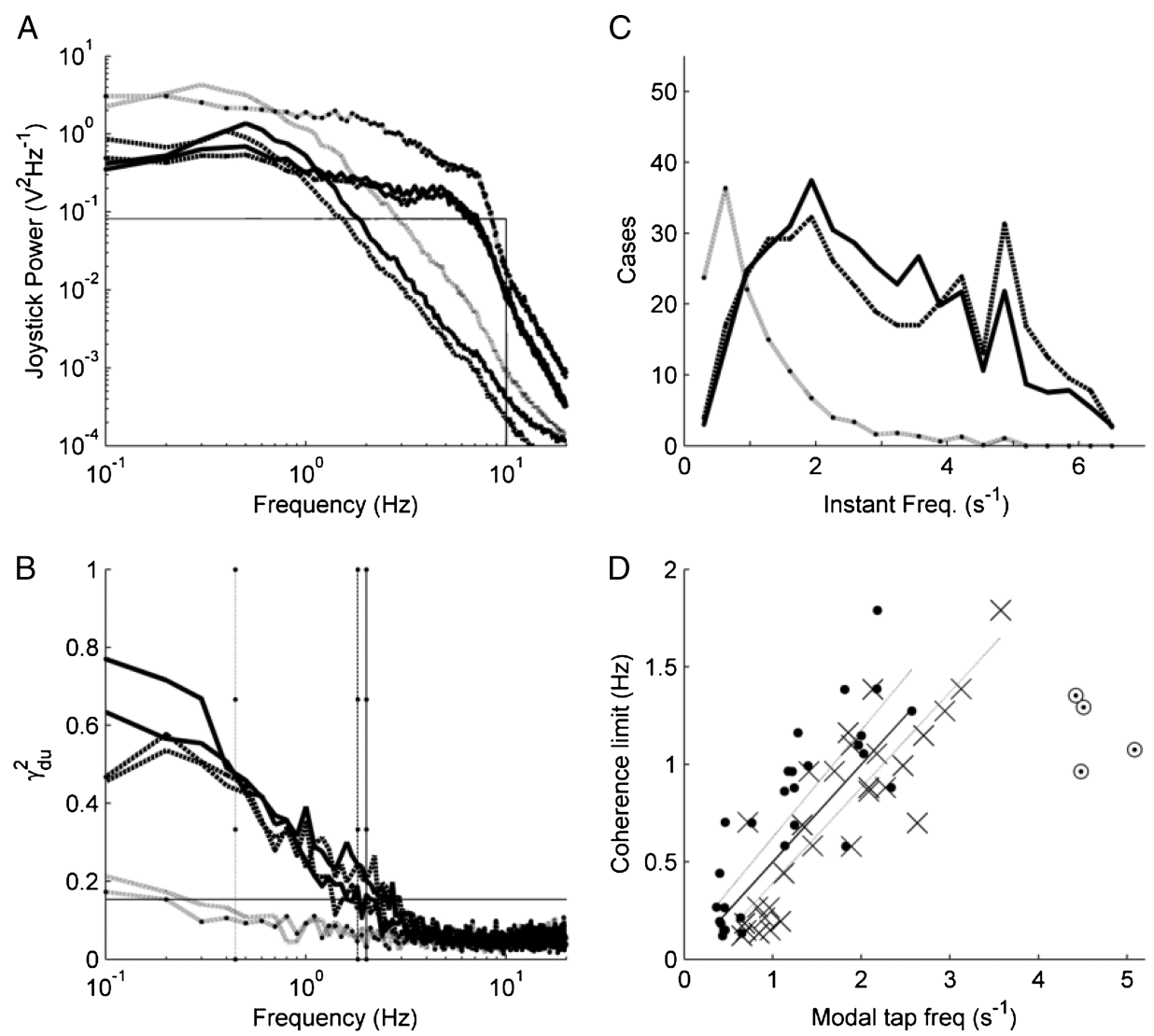

Figure 3. The bandwidth of visuomanual control. This task is the same as Figure 2A except a multi-sine disturbance is added to the joystick signal and there is no tracking target. There are two manual conditions: continuous contact and gentle tapping (dots added to lines). There are three instructions for each condition (position (solid), minimize deviation from center; velocity (broken solid), minimize movement; nonintervention (dotted), wait as long as possible before controlling system). A. Joystick power (curves) and external disturbance power (horizontal line up to $10 \mathrm{~Hz}$ ). B. Coherence ( $\gamma^{2}$ du) between joystick and unpredictable external disturbance; the horizontal line is the value of coherence required for significance at $95 \%$ confidence. C. Incidence of contact during $190 \mathrm{~s}$, binned according to instant frequency (1/contact interval). D. Coherence limit versus modal contact frequency (dots) and versus median contact frequency (crosses) shown for intermittent contact trials from all three goals - position, velocity, nonintervention. Four trials were circled on the grounds that the high-frequency repetitive tapping mode $\left(4-5 \mathrm{~s}^{-1}\right)$ dominated the low-frequency mode at approximately $2 \mathrm{~s}^{-1}$. The solid line shows the predicted relationship (Nyquist sampling frequency) between the coherence limit and modal contact frequency if contact occurred only at the modal frequency. For panels (A) and (B), all curves show variation of the quantities with frequency, for each experimental condition, averaged over 11 trials (one from each subject). For panel (B), vertical lines show the modal contact frequencies, respectively, for tapping trials. [Adapted from (20). Copyright (C) 2011 the authors. Used with permission.]

that is, continuous pathways contribute relatively little power to the overall response (Fig. 1A). This review has summarized our own evidence from a variety of tasks ranging from unperturbed standing balance through pedal and manual control of external mechanical and virtual systems to include whole-body control in a tracking task. Our older evidence supports the serial ballistic hypothesis but does not discriminate against continuous control. The new IC theory and methodology we have developed provide an opportunity to test rigorously this unresolved hypothesis (34). Our new published evidence is limited to tasks with a visual external focus of attention on the results of manual or whole-body control. However, results from these tasks are very clear in demonstrating that continuous control is not necessary (20) and they provide direct evidence of refractoriness in sustained control $(32,33)$. Refractoriness (open-loop intervals) within the feedback loop, as observed in these experiments, is a process missing from the continuous control paradigm (32).

\section{Generalization to Control, Without a Visual External Focus of Attention}

Refractoriness is associated with the serial process of response planning and selection and is known to be amodal $(6,26)$. This process has nothing to do with vision per se. Our experiments, consistent with the intermittent control paradigm, show that refractoriness and, thus, response planning and selection act within the feedback loop. Because refractory response selection samples continuously acquired sensory information intermittently (two to four events per second) for the purpose of constructing control trajectories, this refractory response selection process explains the limited bandwidth $(1-2 \mathrm{~Hz})$ of intentional also known as voluntary control. Thus, intermittent control is appropriate for all motor output below 1 to $2 \mathrm{~Hz}$. The high-bandwidth processes such as spinal, brain stem, and transcortical pathways generate continuous motor responses up to $10 \mathrm{~Hz}$, even higher. For many readers, unperturbed standing balance represents the sustained control task they would most associate with involuntary habitual 


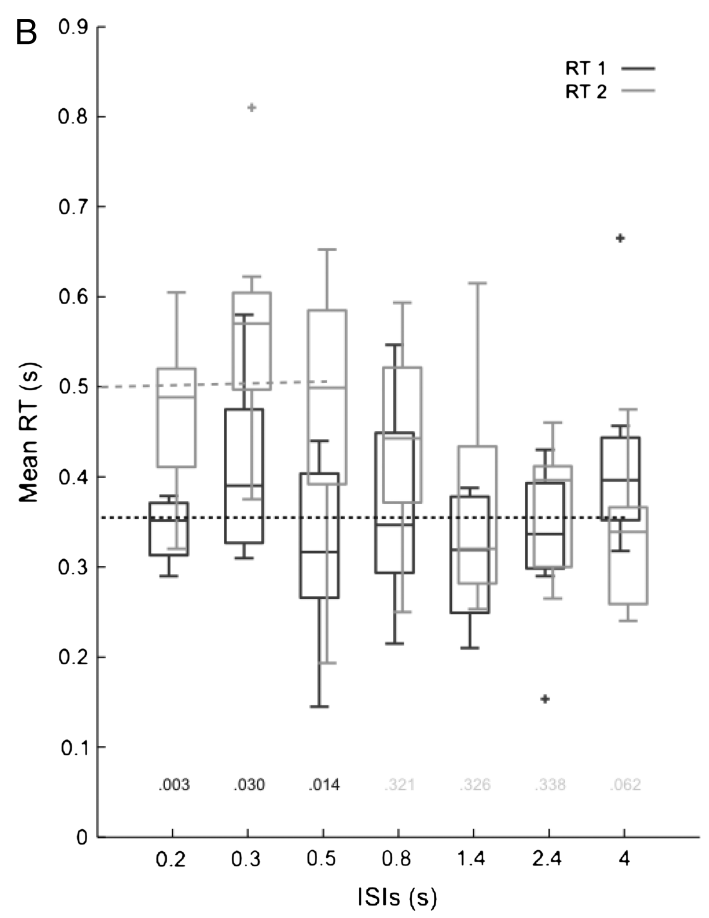

Figure 4. Refractoriness in whole-body control (32). A. The participant receives visual feedback of the Anterior-Posterior head position through a dot presented on an liquid-crystal display (LCD) screen mounted on a trolley. Without moving their feet, participants were asked to track the position of a second dot displayed on the screen. The four possible step sequence combinations (unidirectional and reversed directional step up or down) of the pursuit target are illustrated by the solid line. First and second stimuli are separated by an interstep interval (ISI). The participant experiences an unpredictable sequence of steps. B. Group results. Figure shows the interparticipant mean RT1 (black) and RT2 (gray) against ISI combined across the eight participants. Values of $P$ of the ANOVA post hoc test are displayed above each ISI level (black if $<0.05$, gray if not). The dotted line shows the mean RT1, the dashed line shows the regression linear fit between (interfered) RT2 and ISIs. [Adapted from (32). Copyright (C) 2013 the authors. Used with permission.]

reflexive control. Many would say they stand without thinking about it. For unperturbed standing on a force plate, the position of point of application of the force vector (PoA) economically summarizes the control signal generated by the sole contact force with the external world. From 10 subjects standing unperturbed for $30 \mathrm{~s}$, three times, only $0.36 \% \pm 0.5 \%$ of that signal power $\left(\mathrm{PoA}_{\mathrm{x}}\right.$ anterior posterior component) lies above $2 \mathrm{~Hz}$; thus, we predict that standing balance is regulated mainly by the low-bandwidth intermittent control loop (23).

\section{A General Theory of Motor Control}

As proposed in Figure 1A, a general theory of human control systems must include continuous as well as intermittent processes of which the latter incorporate discrete selection, sampling, and thus switching. For the fast feedback loop (Fig. 1A), continuous systems integrating somatosensory, visual, cutaneous, and vestibular sensory input are well represented by the spinal, brain stem, and transcortical pathways. These pathways provide a high bandwidth feedback at a short latency using feedback parameters that are preselected and open to modulation from outside the pathway by multiple brain regions (27). The neurophysiological basis of systems allowing motor differentiation and choice are less well known. Reflexes are cited traditionally as being "the" primitive system. However, both continuous and switched systems have a primitive basis, which extends through vertebrates (28), invertebrates (2), and even to the level of individual cells. Systems for selecting between multiple possibilities for movement exist within the basal ganglia, prefrontal cortex, and premotor cortex $(4,6,7)$.

For the slow feedback loop, the basal ganglia loops gating the transcortical and subcortical pathways (Fig. 1D) appear to have the correct input, the correct function, and the correct output connections. There is convergence of analyzed sensorimotor input, contextual perceptual and motivational input into and through the basal ganglia (28). Inputs from all major sources, the cerebral cortex, limbic structures, and the thalamus are ordered topographically (28). Basal ganglia outputs contact regions of the thalamus that project back to those regions of cortex providing original inputs (28). Similarly, basal ganglia outputs to the brain stem tend to target those regions that provide indirect input to the basal ganglia. Projections from the basal ganglia output nuclei to the thalamus and brain stem also are topographically ordered. Neurons in the basal ganglia output nuclei have high tonic firing rates $(40-80 \mathrm{~Hz})$. This activity ensures that target regions of the thalamus and brain stem are maintained under a tight and relatively constant inhibitory control. Reduction of inhibitory output releases associated target regions in the thalamus and brain stem from normal inhibitory control $(4,7,28)$. Topologically, in a spiral architecture using successive connections between the limbic, associative, and sensorimotor territories, the basal ganglia are organized to allow selection of overall goal, actions to achieve a selected goal, and submovements to achieve a selected action $(13,28)$. 
The basal ganglia act as a system that dynamically and adaptively gates information flow in transcortical and subcortical pathways $(4,15,28)$. Through hyperdirect, indirect, and direct pathways, this system provides centralized mechanisms for generalized inhibition, specific inhibition, and specific facilitation of action possibilities represented in the frontal cortex $(4,7,28)$. The basal ganglia system does not select directly which action to "consider" but instead modulates the activity of already active representations in cortex (4). This functionality enables cortex to represent multiple potential actions in parallel weakly; the one that first receives a "go" signal from basal ganglia output is then provided with sufficient additional excitation to be executed. Lateral inhibition within the thalamus and cortex acts to suppress competing responses once the winning response has been selected by the basal ganglia circuitry $(4,7)$. The basal ganglia are fundamental to the brain structure of all vertebrates (28). In combination with the prefrontal cortex and premotor cortex, these systems allow low-bandwidth feedback at longer latency using parameters selected online $(3,4,6,7,28)$.

We propose that continuous systems, incorporating muscle spindle and Golgi tendon organ feedback, provide tonic equilibrium joint moments through tonic stretch reflexes, provide partial dynamic stabilization of the unstable mechanical system $(21,25)$, and provide a priming role facilitating intentional feedback. The fast systems alone provides regulation that is highly variable, only partially adequate in rejecting disturbance, and not fully sustained (25). Accurate regulation requires the complete system of high- and lowbandwidth processes acting together. We suggest that the slow IC loop provides central, executive, ongoing regulation of motor output (32) (Fig. 1A). The latency of the first response to a disturbance is determined by the fast system. The frequency bandwidth of sustained control is limited largely by the slower IC loop.

\section{The Rationale for Convergence to a Serial Process Along a Single Channel Within the Feedback Loop}

How reasonable is our hypothesis that motor control tasks are regulated centrally by a serial process along a single channel? While sensory input contains multiple parallel channels and while motor output is executed through multiple muscles acting across multiple joints, the motor system implements concurrently only a small number of task goals. Convergence of goals to few or even one channel is appropriate to optimize coordination (32). Some tasks are incompatible. We cannot flex our knees while extending them. Some tasks are partially compatible, for example, walking and pointing. The selection of compatible routines and the suppression of routines that are partially incompatible or merely inappropriate must underlie skilled and economical task performance. Optimization of coordination of tasks by eliminating mutual interference - in effect becomes the same thing as controlling a single task in the task space. Hence, we offer the rationale that optimization of coordination leads to unification of tasks into a single channel for its control.

A single output at task level can be implemented by lowerlevel continuous feedback systems. Optimal control provides solutions for distributing a single task across multiple redundant motor systems $(27,31)$. However, the processes of planning, optimization, and selecting the single-channel output should occur within the main feedback loop when these processes require the current system state as input (30). If these processes require computational time, then time has to elapse between sampling the system state and producing the selected motor output. If successful optimization and implementation of this single selection require temporary inhibition of competing possibilities, then refractoriness is required. This rationale justifies open-loop intermittent feedback control (30). Computing solutions as required, avoids the necessity task of storing motor solutions for every eventuality. At a cost of reduced control bandwidth (e.g., 1-2 Hz), intermittent control maximizes online flexibility and adaptability $(32,33)$.

\section{Clarification of Terms Related to Intermittent}

In presenting the hypothesis for intermittent control, we are aware that the ambiguity of some terms (e.g., intentional, voluntary, planning, selecting) may lead the reader to the wrong idea with respect to consciousness, verbalization, and decision making. The tasks for which we have direct evidence of refractoriness required attention to the task goal. One is not necessarily conscious of the process underlying control of the hand or body, although some participants may have more awareness than others of what they did. Some participants are very practiced in these tasks such that to the participant control appears to proceed automatically provided one is attending to the task. There is no verbal component, and participants are not necessarily able to describe what they have done. Whatever decision making, selection, or processing is involved, this control is best described as implicit. We call this control intentional on the basis of the feedback delay (180-230 ms), the level of flexibility/response choice facilitated (22), and the fact that the processing and selection are online (i.e., within the feedback loop) rather than offline (preselected). "Intentional" refers to the flexibility of control in the moment rather than one's awareness of that flexibility.

\section{CONCLUSIONS}

We challenge the prevailing idea that human motor control is explained most powerfully and accurately by the continuous paradigm (27) and by continuous neurophysiological mechanisms (27). Using new theoretical development, methodology, and new evidence of refractoriness during sustained control, we propose that intermittent control, which incorporates a serial ballistic process within a slow feedback loop, provides the main regulation of motor effort, supplemented by fast, lower-level, continuous feedback. Refractoriness distinguishes the slow intentional from the fast reflexive loop. IC in which optimization and selection occur within the feedback loop provides powerful advantages for performance and survival. A potential neurophysiological basis for IC lies in centralized selection and optimization pathways including, respectively, the basal ganglia and cerebellum (15). 


\section{Acknowledgments}

The authors acknowledge the work of other researchers that could not be cited because of reference limitation. Authors we are aware of contributing directly to ideas of intermittent and serial ballistic control include Craik (1947-1948), Vince (1948), Navas and Stark (1968), Beggs (1974), Poulton (1974), Miall et al. (1986), Neilson (1988-2012), Miall and Wolpert (1992-3), Hanneton et al. (1997), Slifkin and Newell (2000-), Russell (2001), Gross (2002), Morasso (1999-), Milton (2005-), Botarro et al. (2005-), Fishbach (2005-), Stepan and Insperger (2006-), Houk (2007-), Asai (2009-), Suszuki (2012-), and Karniel (2013). The theory of intermittent control and its application to human motion control have been developed during the past 25 years by P.D. Neilson and M.D. Neilson. P. Gawthrop acknowledges discussions with them about intermittent control during the past 14 years. The authors acknowledge EPSRC financial support for this work via the linked grants EP/F068514/1, EP/F069022/1, and EP/F06974X/1.

\section{References}

1. Bernstein NA, Latash LL, Turvey MT. On Dexterity and Its Development. Mahwah, NJ: Lawrence Erlbaum Associates; 1996:3.

2. Brembs B. Towards a scientific concept of free will as a biological trait: spontaneous actions and decision-making in invertebrates. Proc. Royal Soc. B. Biol. Sci. 2010;278:930-939.

3. Cisek P, Kalaska JF. Neural correlates of reaching decisions in dorsal premotor cortex: Specification of multiple direction choices and final selection of action. Neuron. 2005; 45(5):801-14.

4. Cohen MX, Frank MJ. Neurocomputational models of basal ganglia function in learning, memory and choice. Behav. Brain Res. 2009; 199(1):141-56.

5. Diedrichsen J, Shadmehr R, Ivry RB. The coordination of movement: optimal feedback control and beyond. Trends Cogn. Sci. 2010; 14(1):31-9.

6. Dux PE, Ivanoff J, Asplund CL, Marois R. Isolation of a central bottleneck of information processing with time-resolved fMRI. Neuron. 2006; 52(6):1109-20.

7. Frank MJ. Computational models of motivated action selection in corticostriatal circuits. Curr. Opin. Neurobiol. 2011; 21(3):381-6.

8. Gawthrop P, Loram I, Lakie M, Gollee H. Intermittent control: a computational theory of human control. Biol. Cybern. 2011; 104(1-2):31-51.

9. Gawthrop P, Wang L. The system-matched hold and the intermittent control separation principle. Int. J. Control. 2011; 84(12):1965-74.

10. Gawthrop PJ. Frequency-domain analysis of intermittent control. Proc. Inst. Mech. Eng. 2009; 223(I5):591-603.

11. Gawthrop PJ, Wang L. Event-driven intermittent control. Int. J. Control. 2009; 82(12):2235-48.

12. Gollee H, Mamma A, Loram I, Gawthrop P. Frequency-domain identification of the human controller. Biol. Cybern. 2012; 106(6): 359-72.

13. Haber SN, Fudge JL, McFarland NR. Striatonigrostriatal pathways in primates form an ascending spiral from the shell to the dorsolateral striatum. J. Neurosci. 2000; 20(6):2369-82.

14. Hardwick RM, Rottschy C, Miall RC, Eickhoff SB. A quantitative metaanalysis and review of motor learning in the human brain. Neurolmage. 2013; 67:283-97.
15. Houk JC, Bastianen C, Fansler D, et al. Action selection and refinement in subcortical loops through basal ganglia and cerebellum. Philos. Trans. Royal Soc. B. Biol. Sci. 2007; 362(1485):1573-83.

16. Kleinman DL, Baron S, Levison WH. An optimal control model of human response 1. Theory and validation. Automatica. 1970; 6(3):357-69.

17. Lakie M, Loram ID. Manually controlled human balancing using visual, vestibular and proprioceptive senses involves a common, low frequency neural process. J. Physiol. 2006; 577(Pt 1):403-16.

18. Levison WH, Baron S, Kleinman DL. A model for human controller remnant. Man-Machine Syst. IEEE Trans. 1969; 10(4):101-8.

19. Loram ID, Gawthrop PJ, Lakie M. The frequency of human, manual adjustments in balancing an inverted pendulum is constrained by intrinsic physiological factors. J. Physiol. (Lond.). 2006; 577(1):417-32.

20. Loram ID, Gollee H, Lakie M, Gawthrop PJ. Human control of an inverted pendulum: is continuous control necessary? Is intermittent control effective? Is intermittent control physiological? J. Physiol. (Lond.). 2011; 589(2):307-24.

21. Loram ID, Lakie M. Human balancing of an inverted pendulum: position control by small, ballistic-like, throw and catch movements. J. Physiol. 2002; 540(Pt 3):1111-24.

22. Loram ID, Lakie M, Gawthrop PJ. Visual control of stable and unstable loads: what is the feedback delay and extent of linear time-invariant control? J. Physiol. 2009; 587(Pt 6):1343-65.

23. Loram ID, Maganaris CN, Lakie M. Human postural sway results from frequent, ballistic bias impulses by soleus and gastrocnemius. J. Physiol. 2005; 564(Pt 1):295-311.

24. Loram ID, van de Kamp C, Gollee H, Gawthrop PJ. Identification of intermittent control in man and machine. J. Royal Soc. Interf. 2012; 9(74): 2070-84.

25. Marsden CD, Merton PA, Morton HB, Rothwell JC, Traub MM. Reliability and efficacy of the long-latency stretch reflex in the human thumb. J. Physiol. (Lond.). 1981; 316:47-60.

26. Pashler H, Johnston JC, Pashler H. Attentional Limitations in Dual-Task Performance. Hove: Psychology Press; 1998:155.

27. Pruszynski JA, Scott SH. Optimal feedback control and the long-latency stretch response. Exp. Brain Res. 2012; 218(3):341-59.

28. Redgrave P. Basal ganglia. J. Scholarpedia. 2007; 2(6):1825.

29. Redgrave P, Gurney K. The short-latency dopamine signal: a role in discovering novel actions? Nat. Rev. Neurosci. 2006; 7(12):967-75.

30. Ronco E, Arsan T, Gawthrop PJ. Open-loop intermittent feedback control: Practical continuous- time GPC. IEE Proc. Control Theory Appl. 1999; 146(5):426-34.

31. Todorov E. Optimality principles in sensorimotor control. Nat. Neurosci. 2004; 7(9):907-15.

32. Van De Kamp C, Gawthrop P, Gollee H, Lakie M, Loram ID. Interfacing sensory input with motor output: does the control architecture converge to a serial process along a single channel? Front Comput. Neurosci. 2013; 7:55.

33. Van de Kamp C, Gawthrop PJ, Gollee H, Loram ID. Refractoriness in sustained visuo-manual control: is the refractory duration intrinsic or does it depend on external system properties? PLoS Comput. Biol. 2013; 9(1): e1002843.

34. Vince MA. The intermittency of control movements and the psychological refractory period. Br. J. Psychol. 1948; 38(3):149-57.

35. Yin HH, Knowlton BJ. The role of the basal ganglia in habit formation. Nat. Rev. Neurosci. 2006; 7(6):464-76. 